\title{
Universal spectral dynamics of modulation instability: theory, simulation, experiment
}

\author{
K. Hammani ${ }^{1}$, B. Wetzel ${ }^{2}$, B. Kibler ${ }^{1}$, J. Fatome ${ }^{1}$, C. Finot ${ }^{1}$, \\ N. Akhmediev' ${ }^{3}$ J. M. Dudley ${ }^{2}$ \\ 1. Laboratoire Interdisciplinaire Carnot de Bourgogne, Université de Bourgogne, 21078 Dijon, France \\ 2. Institut FEMTO-ST, Université de Franche-Comté, 25030 Besançon, France \\ 3 Optical Sciences Group, Research School of Physics and Engineering, Institute of Advanced Studies, The \\ Australian National University, Canberra ACT 0200, Australia
}

A central process of nonlinear fibre optics is modulation instability (MI), where weak perturbations on a continuous wave are amplified to generate a parametric cascade of spectral sidebands. Although studied for many years, it has only been recently appreciated that MI dynamics can be described analytically by Akhmediev breather (AB) solutions to the nonlinear Schrödinger equation (NLSE) [1]. This has led to important results, including the first observation of the Peregrine Soliton [2]. AB theory has also shown that the spectral amplitudes at the peak of the MI gain curve yield a characteristic log-triangular spectrum, providing new insight into the initial phase of supercontinuum generation [3]. Here, we present a significant extension to this theory by generalising the analysis to describe spectral characteristics for arbitrary gain. Our new result also describes the dynamics of the spectral development, a process previously studied only via truncated models or numerical approaches [4]. We confirm our theory by simulations and experiments.

Our analysis is based on a fibre NLSE system with the usual dispersion and nonlinearity parameters $\beta_{2}$ and $\gamma$. For initial plane wave power $\mathrm{P}_{0}$, the $\mathrm{AB}$ solution describes field evolution as a function of a variable independent frequency $\omega_{\mathrm{mod}}$ in the range $\omega_{\mathrm{c}}>\omega_{\mathrm{mod}}>0$ where $\omega_{\mathrm{c}}^{2}=4 \gamma P_{0} /\left|\beta_{2}\right|$. With $2 a=\left[1-\left(\omega_{\mathrm{mod}} / \omega_{\mathrm{c}}\right)^{2}\right], b=[8 a(1-2 a)]^{1 / 2}$ and $p=\sqrt{2}$ $\omega_{\text {mod }} / \omega_{\mathrm{c}}$, the normalised spectral amplitudes of the pump $\mathrm{f}_{0}$ and $\mathrm{n}$-th sideband $\mathrm{f}_{\mathrm{n}}$ evolve with distance as follows:

$$
f_{0}(\xi)=1-\frac{i b \sinh b \xi+p^{2} \cosh b \xi}{\sqrt{\cosh ^{2} b \xi-2 a}}, \quad f_{n}(\xi)=\frac{i b \sinh b \xi+p^{2} \cosh b \xi}{\sqrt{\cosh ^{2} b \xi-2 a}}\left[\frac{\cosh b \xi-\sqrt{\cosh ^{2} b \xi-2 a}}{\sqrt{2 a}}\right]^{|n|}
$$

Here distance $\xi=\mathrm{z} / \mathrm{L}_{\mathrm{NL}}$ with $\mathrm{L}_{\mathrm{NL}}=\left(\gamma \mathrm{P}_{0}\right)^{-1}, \mathrm{n}= \pm 1, \pm 2$ etc. and we do not write constant amplitude and phase factors. This result has been confirmed experimentally by studying induced MI under a wide range of conditions around $1550 \mathrm{~nm}$ (we choose parameters far from the gain peak where $\mathrm{a}=0.25$ ). In one experiment using highly nonlinear fibre and $\omega_{\bmod } / 2 \pi=241 \mathrm{GHz}$ such that a $=0.42$, Fig. 1(a) compares sideband amplitudes from experiment and theory at the point where there is maximum breather localization. We also show results from simulations (including Raman, loss etc) and we see excellent agreement between experiment, theory and simulation. In another experiment using standard telecommunications-grade fibre and $\omega_{\text {mod }} / 2 \pi=16 \mathrm{GHz}$ such that $\mathrm{a}=0.45$, cutback measurements allowed direct measurement of evolution with distance. These results in Fig. 1(b) show evolution of pump (left) and first sideband amplitude (right) and we again see good agreement between experiment, theory and simulation. Discrepancies are attributed to differences in assumed initial conditions between theory and experiment.

These results provide important confirmation of this new analytic result describing $\mathrm{AB}$ evolution. In addition to their fundamental extension of the theory of nonlinear MI propagation, we also anticipate important consequences of these results in designing optimized setups for nonlinear pulse train generation under arbitrary conditions.

(a) Point of Maximum Localisation

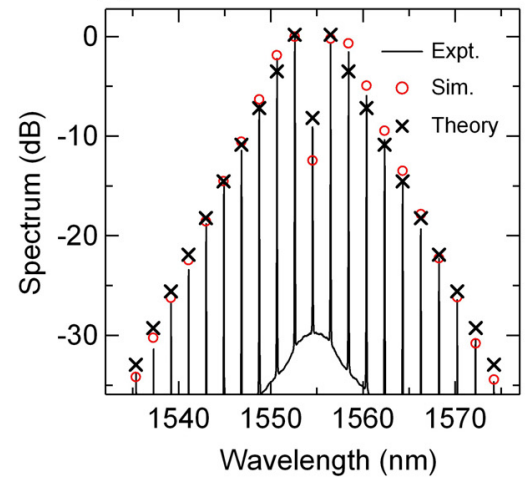

(b) Dynamics with Propagation Distance
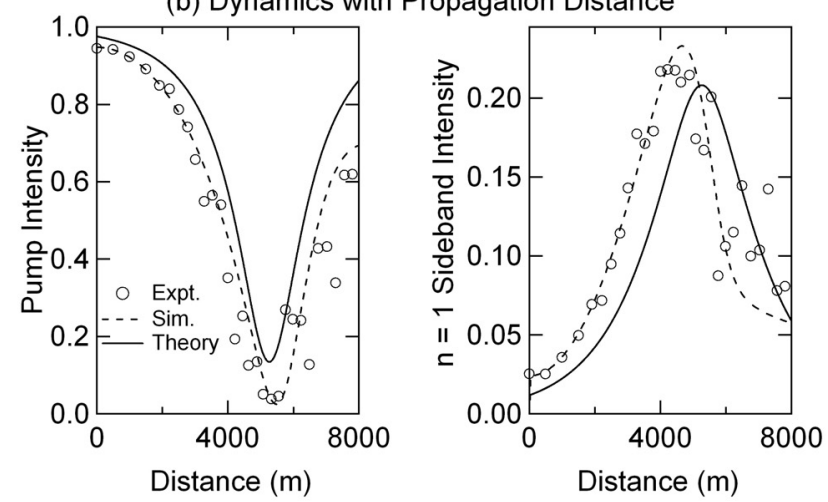

Fig. 1 (a) MI spectrum at point of maximum localisation for $a=0.42$. (b) Pump and sideband dynamics for $a=0.45$.

\section{References}

1. N. Akhmediev, V. I. Korneev, Theor. Math. Phys. 69, 1089-1093 (1986)

2. B. Kibler et al. Nature Phys. 6, 790-795 (2010); K. Hammani et al. Opt. Lett. in press, publ. online ID 137339 (2011)

3. J. M. Dudley, G. Genty, F. Dias, B. Kibler, N. Akhmediev, Opt. Express 17, 21497-21508 (2009)

4. G. Van Simaeys, Ph. Emplit, M. Haelterman, Phys. Rev. Lett. 87, 033902 (2001) 